\title{
The Medical Birth Registry of Norway - An international perspective
}

\author{
Allen J. Wilcox \\ Epidemiology Branch, National Institute of Environmental Health Sciences, PO Box 12233, Durham, NC 27709, USA \\ Telephone: +1-919-541-4660 Telefax:+1-919-541-2511 E-mail: wilcox@niehs.nih.gov
}

\begin{abstract}
Some of the most practical questions of perinatal medicine are regarding couples who have had pregnancy problems in the past, and their risk of having such problems in future pregnancies. For example, if a couple has a child with a birth defect, what are their chances that their next child will have a defect? The key to answering such questions is the availability of linked data such as those provided by the Medical Birth Registry of Norway. Such linked data provide a unique resource for addressing a broad range of questions in perinatal epidemiology. The Medical Birth Registry of Norway has been a pioneer in answering such questions.
\end{abstract}

If a mother has a stillborn baby, what are her chances of having a healthy baby at her next pregnancy? If a father has a birth defect, is his own child likely to have the same birth defect? Is his child at risk for other kinds of birth defects?

These are concrete and practical concerns of parents. Even though most pregnancies produce healthy babies, at least a third of all couples have one or more pregnancies with problems. These problems can range from miscarriage to preterm delivery to an offspring with a malformation. It is natural for couples to have some worries when they become pregnant. Couples who have had difficulty in a past pregnancy are likely to be even more concerned.

What assurances can researchers provide? The questions are simple, but the answers have been surprisingly difficult to generate. Data from whole populations are the gold standard for answering such questions. It is only relatively recently that epidemiologists have had population-based data on the recurrence of pregnancy problems. But population-based data by themselves are still not enough.

\section{THE IMPORTANCE OF LINKED DATA}

The key to addressing this problem is the availability of linked data. By "linked", epidemiologists mean a birth record that can be connected to other births from the same woman, or to other records for the same baby. To appreciate the importance of linked data, we should first consider unlinked birth data. Much of what we know about infant mortality has come from birth certificates collected as part of vital statistics. Most countries have laws that require collection of vital statistics, including legal records of births and deaths. These birth certificates typically exist in isolation, without being linkable to other deliveries by the same woman, or to later health problems occurring to that baby.
Without linkage, it is difficult or impossible to answer the kinds of questions raised above. For example, to estimate the chances of a future healthy pregnancy among mothers who have had a stillbirth, we would need records from a large number of mothers who have had a stillbirth, and from a comparison group of mothers who have had a healthy pregnancy. Then for each mother, past records would have to be linked with subsequent pregnancies, so that the risk at later pregnancy could be calculated for the two groups of women.

Such linkage is rare. There may be legal barriers for reasons of confidentiality. If linkage is permissible, it may not be feasible. Birth certificates routinely contain parents' names but no personal identification numbers. Many records can of course be linked by mother's name alone, but there are many more that cannot: common names are shared by different mothers, or women change their names, or women move from one registration district to another. The Scandinavian countries are among the few places in the world in which all births from a particular mother and father can be systematically linked. Norway was the first to do so.

The Medical Birth Registry of Norway has been a pioneer among the linked registries, providing linkages through the unique personal identification number assigned to every person at birth. This resource has allowed Norway to become a world leader in providing information to parents about the risk of problems in future pregnancies.

\section{THE USES OF LINKED DATA}

This capacity to link birth records for a whole nation helped bring the Medical Birth Registry of Norway to international attention. Within ten years of the start of the Registry in 1967, enough women had delivered two or more pregnancies for Norwegian researchers to begin to analyze these linked pregnancies. The first 
scientific report based on linkage was published in 1977 by Leiv Bakketeig, showing that mothers who had one baby born preterm or low birth weight were at increased risk of having another. ${ }^{1}$

This was the beginning of a stream of scientific papers that have described risk in future pregnancies for couples who have had a poor outcome. As pregnancies accumulated in the Birth Registry, researchers were able to describe risks with more specific outcomes. In 1984, Lorenz Irgens and his colleagues published data showing that the relative risk of recurrence for sudden infant death syndrome (or cot death) was 3.7, much lower than the ten-fold increase estimated previously by studies based on more limited samples. ${ }^{2}$ In 1994, Rolv Terje Lie and his colleagues looked at risk of birth defects among couples whose first baby had a birth defect. ${ }^{3}$ On average, such couples had eight times the background risk of having the same birth defect in their second child. However, this risk was very small in absolute terms - only a few percent of second babies were affected with the same birth defect. Furthermore, the couples' risk of having a baby with any other type of defect was not much different than for other couples. Thus, among couples who had one affected child, the vast majority - around 95\% - could expect their next baby to be free of any recognized malformations at birth.

\section{LINKAGE BETWEEN REGISTRIES}

Researchers soon recognized that they could link births from the Medical Birth Registry with later health outcomes recorded in other Registries. Thus, in 1985, Gayle Windham and her colleagues explored whether babies with birth defects are at higher risk for childhood cancers (they are not). ${ }^{4}$ Researchers have also been able to consider whether a woman's pregnancies affects her own risk of later disease. Lars Vatten and his colleagues showed that a woman with a preeclamptic pregnancy has a lower risk of breast cancer than other women, for reasons not yet understood. $^{5}$

One of the most influential papers in this regard was by Henrik Irgens and his colleagues. ${ }^{6}$ These investigators assessed the later cardiovascular mortality of women who had had a preeclamptic pregnancy. They found that the risk of mortality from cardiovascular disease was increased eight-fold among women who had had a premature baby from a preeclamptic pregnancy. This finding has generated interest in the link between preeclampsia and heart disease, and suggests that preeclampsia may be an early expression of a woman's risk of heart disease.

\section{TWO-GENERATION STUDIES}

By the mid-1990s, yet another opportunity began to unfold: persons born into the Registry were becoming old enough to have children of their own. This allowed a new type of study that assessed familial risk across generations. In two widely-cited papers, ${ }^{7,8}$ Norwegian investigators described the chances that babies with a birth defect would grow up to have offspring with birth defects. Once again, the results were reassuring. While the affected parents had an increased risk of the same defect in their own baby, this risk was very small in absolute terms. Overall, $95 \%$ of the babies born to affected fathers or affected mothers had no birth defect recorded in the Registry.

\section{SURVEILLANCE}

One of the original purposes of the Medical Birth Registry was to establish a tool for surveillance, a tool that could be used to identify newly emerging risks. Surveillance tools are useful because they can address questions that were not even imagined at the outset. For example, the Chernobyl accident in 1986 exposed Norwegians to radiation through direct airborne contact and through contamination of local foods. There was understandable concern about possible health effects including birth defects. An analysis of the rate of birth defects before and after the accident showed no increase in any of the types of birth defects associated with radiation exposure. ${ }^{9}$ Such assurance would have been impossible if the Registry had not been established and in full operation before the accident occurred.

\section{THE INTERNATIONAL IMPACT OF THE MEDICAL BIRTH REGISTRY OF NORWAY}

In 1995, the US Centers for Disease Control and the US National Institutes of Health organized an international symposium on maternally-linked pregnancy outcomes. Along with the excellent linked Registries of Sweden and Denmark, the Medical Birth Registry of Norway was one of the centerpieces of that symposium. ${ }^{10}$ Ten years later, in 2005, a second international symposium was held, and once again, Norwegian researchers played a prominent role. ${ }^{11,12}$

Over the past 40 years - and especially in the past 20 years - the Medical Birth Registry of Norway has made major scientific contributions to medicine and public health. During this time, more than 20 papers have been published in the leading weekly clinical journals - the most influential journals in the field of medicine and public health. Thirty papers have been published in the international specialty clinical journals in pediatrics, obstetrics and other fields, and nearly 80 papers have appeared in major epidemiology journals. This prodigious scientific productivity is a credit to the researchers of Norway, and most especially to the founders of the Medical Birth Registry. The founders had faith that the seeds they planted would contribute to the health of the Norwegian people. What the founders could not have foreseen was the benefits that the Registry would provide to the world at large. 


\section{REFERENCES}

1. Bakketeig LS. The risk of repeated preterm or low birth weight delivery. In: Reed DM, Stanley FJ, eds. The Epidemiology of Prematurity. Baltimore: Urban and Schwarzenberg, 1977: 231-41.

2. Irgens LM, Skjaerven R, Peterson DR. Prospective assessment of recurrence risk in sudden infant death syndrome siblings. J Pediatr 1984; 104: 349-51.

3. Lie RT, Wilcox AJ, Skjærven R. A population-based study of risk of recurrence of birth defects. New Engl J Med 1994; 331: 1-4.

4. Windham GC, Bjerkedal T, Langmark F. A population-based study of cancer incidence in twins and in children with congenital malformations or low birth weight, Norway, 1967-1980. Am J Epidemiol 1985; 121 (1): 49-56.

5. Vatten LJ, Romundstad PR, Trichopoulos D, Skjaerven R. Pre-eclampsia in pregnancy and subsequent risk for breast cancer. Br J Cancer 2002; 87 (9): 971-3.

6. Irgens HU, Reisaeter L, Irgens LM, Lie RT. Long term mortality of mothers and fathers after pre-eclampsia: population based cohort study. BMJ 2001; 323 (7323): 1213-7.

7. Skjærven R, Wilcox AJ, Lie RT. A population-based study of survival and childbearing in women with birth defects, and risk of recurrence in offspring. New Engl J Med 1999; 340: 1057-62.

8. Lie RT, Wilcox AJ, Skjaerven R. Survival and reproduction in males with birth defects. JAMA 2001; 285: $755-60$.

9. Lie RT, Irgens LM, Skjaerven R, Reitan JB, Strand P, Strand T. Birth defects in Norway by levels of external and food-based exposure to radiation from Chernobyl. Am J Epidemiol 1992; 136 (4): 377-88.

10. Herman AA, McCarthy BJ, Bakewell JM, Ward RH, Mueller BA, Maconochie NE, Read AW, Zadka P, Skjaerven R. Data linkage methods used in maternally-linked birth and infant death surveillance data sets from the United States (Georgia, Missouri, Utah and Washington State), Israel, Norway, Scotland and Western Australia. Paediatr Perinat Epidemiol 1997; 11 (Suppl 1): 5-22.

11. Ananth CV. Second International Symposium on Successive Pregnancy Outcomes: a Decade of Progress. Paediatr Perinat Epidemiol 2007; 21 (Suppl 1): 1-3.

12. Lie RT. Intergenerational exchange and perinatal risks: a note on interpretation of generational recurrence risks. Paediatr Perinat Epidemiol 2007; 21 (Suppl 1): 13-18. 